\title{
Métodos de enseñanza del dibujo de observación en la graduación de diseño
}

\author{
Methods of teaching of observation drawing in design graduation \\ Métodos de ensino do desenho de observação na graduação de design \\ Luiz Carlos Teixeira da Silva', Milton Koji Nakata² \\ ${ }^{1}$ Graduado en Diseño Industrial, Faculdade de Ciências e Tecnologia de Birigui. Maestrando en Design, \\ PPG Design. Universidade Estadual Paulista Júlio de Mesquita Filho, UNESP, Bauru-SP, Brasil. Profesor \\ Faculdade de Ciências e Tecnologia de Birigui, FATEB. Email: luiz@doisdi.com \\ 2 Graduado en Comunicación Visual, Fundação Educacional de Bauru, maestria em Projeto Arte e \\ Sociedade, Universidade Estadual Paulista Júlio de Mesquita Filho. Doctorado en Comunicação e Poéticas \\ Visuais, Universidade Estadual Paulista Júlio de Mesquita Filho. Profesor asistente doctor Universidade \\ Estadual Paulista
}

Recibido: 9 de agosto 2017. Aceptado: 10 de octubre de 2017.

Teixeira, L.; Nakata, M. (2017). Métodos de enseñanza del dibujo de observación en la graduación de diseño. Procesos urbanos Número 4, Ene-Dic. 149-156. DOI:dx.doi.org/10.21892/2422085X.357

\section{RESUMEN}

Este trabajo busca presentar una deficiencia que afecta a profesionales que trabajan con el dibujo, como los de diseño, arquitectura y moda. Debido al distanciamiento del ejercicio del dibujo que suele ocurrir durante la infancia, alumnos de cursos superiores suelen encontrarse en una situación crítica al retomar la práctica. Afortunadamente, se cree que ciertos métodos pueden mejorar el rendimiento de los alumnos en la producción del dibujo. El texto presenta datos sobre una experiencia con dibujo de observación y relata datos parciales de una investigación de maestría que busca evaluar la influencia de métodos de ayuda en la observación y en la ejecución de dibujos.

Palabras clave: dibujo; metodología; enseñanza.

\section{ABSTRACT}

This work seeks to present a deficiency that affects professionals working with the drawing, such as design, architecture and fashion. Due to the distancing of the exercise of drawing that usually occurs during childhood, students of higher education usually find themselves in a critical situation when resuming the practice. Fortunately, it is believed that certain methods can improve student performance in drawing production. The text presents data about an experiment with observation drawing and reports partial data from a master's research that seeks to evaluate the influence of aid methods on the observation and execution of drawings.

Keywords: drawing, methodology, teaching. 


\section{RESUMO}

Esse trabalho procura apresentar uma deficiência que atinge profissionais que trabalham com o desenho, como os de design, arquitetura e moda. Devido ao distanciamento do exercício do desenho que costuma ocorrer durante a infância, alunos de cursos superiores costumam se encontrar em uma situação crítica ao retomar a prática. Felizmente, acredita-se que determinados métodos podem melhorar o desempenho dos alunos na produção do desenho. O texto apresenta dados sobre uma experiência com desenho de observação e relata dados parciais de uma pesquisa de mestrado que procura avaliar a influência de métodos de auxílio na observação e na execução de desenhos.

Palavras chave: desenho, metodologia, ensino.

\section{Introdução}

O desenho e sua prática estão presentes em nossas vidas desde a infância e nos expressamos através dele. Mesmo fora do ambiente escolar, o indivíduo utiliza - desenho como linguagem, desde seu processo de aprendizagem, retratando ideias e mensagens através de diversas formas de representação gráfica. Infelizmente, ainda na vida escolar, a maioria dos alunos começa um processo de distanciamento da prática de desenhar. Isso parece ser parte da cultura educacional, que mantém as artes em um grau inferior de importância.

Essa deficiência se torna ainda mais crítica quando afeta alunos de cursos superiores onde a prática do desenho é essencial, como acontece em cursos superiores como os de design, arquitetura e moda. Distantes da prática do desenho e acostumados com outras formas de representação, esses indivíduos sofrem com a retomada da atividade perdida na infância. Essa dificuldade e um aparente desinteresse pelo desenho feito à mão estão desvalorizando a principal ferramenta para produzir e transmitir ideias.

Durante a graduação, esses alunos encaram diversas atividades projetuais que dependem da capacidade criativa, da geração de ideias e da apresentação de propostas através de desenhos. Nesse cenário, se instaura também uma dificuldade em esclarecer aos alunos que o desenho é vital para sua vida profissional. Afinal, sem o desenho como meio de reflexão projetual, não seria possível a produção de projetos inovadores e de sucesso. "O desenho como projeto coloca à prova as hipóteses, transformandoas em predições verificáveis" (Massironi, 2015. p. 10). A falta de empenho no ensino e na prática de desenhar, assim como as dificuldades próprias de cada aluno de curso superior de design, cria um cenário negativo. "Jovens profissionais saem para o mercado de trabalho com pouca capacidade representativa e com um portfólio pobre de trabalhos visuais" (Nakata, 2011. p. 15). Felizmente, existem métodos que podem melhorar o desempenho dos alunos na prática do desenho, assim como esclarecem alguns autores.

Como algumas habilidades globais - por exemplo, ler, dirigir, esquiar e andar -, desenhar compõe-se de outras habilidades parciais que se integram numa habilidade total. Uma vez aprendidos e integrados os componentes, você conseguirá desenhar - assim como uma vez aprendida a habilidade de ler, sabe-se ler por toda a vida; uma vez que se aprende a andar, sabe-se andar por toda a vida (Edwards, 2002. p. 18).

Métodos podem ser aplicados dentro de algumas disciplinas, como naquelas que abordam geometria, desenho técnico, métodos e linguagens visuais e desenho de observação. Essas disciplinas trabalham conceitos de técnicas de desenho, representação, linguagem visual e outros aspectos que são essenciais na educação do desenho.

Esse trabalho apresenta informações levantadas durante a ministração de aulas de desenho de observação para alunos 
matriculados em um curso superior de Design. Além disso, no capítulo seguinte, descreve parte de uma pesquisa de mestrado que avalia métodos e técnicas para o ensino de desenho. $O$ ato de desenhar abordado aqui se restringe ao manual (ou analógico) e à retratação do real pela observação e não se relaciona ao trabalho produzido pelo desenho criativo, imaginativo e expressivo ou mesmo classificado como artístico. Com essas informações, objetiva-se contribuir no ensino do desenho e na qualidade dos trabalhos produzidos por alunos de design.

\section{Um relato sobre a disciplina de desenho de observação}

Uma disciplina de desenho de observação para cursos como o de design e de arquitetura costuma ter como objetivo desenvolver - senso de observação, criatividade e a habilidade manual para representação de imagens. Tais diretrizes conduzem o que docente deve apresentar aos alunos, na definição do conteúdo da disciplina e no modo como os exercícios devem ser trabalhados. $O$ docente deve ainda evidenciar a importância do desenho como ferramenta essencial nas atividades profissionais. Segundo Jenny (2014, p. 13), "precisamos salientar que desenhar não é apenas uma arte, mas também uma disciplina intelectual".

Os dados apresentados nesse capítulo descrevem um trabalho realizado no ano de 2015, com uma turma de treze alunos matriculados em curso de bacharel em design de uma instituição municipal brasileira, localizada no interior do estado de São Paulo. É pertinente relatar que tal instituição não aplica em seu processo seletivo uma avaliação de aptidão para desenho, como costuma ser aplicada por algumas instituições. Desse modo, apesar do interesse dos candidatos pela área escolhida, a maior parte dos ingressantes possui um nível baixo de habilidade para o desenho. É importante citar aqui que todos os treze alunos já tinham alguma experiência própria com desenho ou interesse pela prática, no entanto não haviam realizado anteriormente nenhum curso de desenho e se consideravam autodidatas ou incapazes de desenhar. A disciplina intitulada como
Desenho de Observação foi ministrada no segundo semestre do curso e, observando esse perfil característico de alunos, foi necessário aplicar a teoria e a prática nos níveis mais básicos, pelo menos no início do semestre.

Foi desenvolvido então um plano de ações para que a teoria e as atividades práticas conduzissem os alunos para um nivel de aptidão no desenho superior ao que eles apresentavam no início do semestre. A primeira ação objetivou avaliar o nível de representação e foi solicitado aos alunos que desenhassem um pertence utilizando suas capacidades atuais de representação. Tal objeto seria desenhado novamente no final do semestre como atividade final da disciplina. Conscientes do processo, os desenhos foram produzidos pelos alunos que utilizaram lápis grafite em uma folha de sulfite no tamanho A4, tamanho que foi adotado para todas as atividades seguintes.

Nas semanas seguintes, as atividades foram conduzidas de acordo com o nível de aptidão dos alunos observado pelo docente. As aulas apresentavam uma determinada teoria, seguida de pequenos exercícios práticos para estimular a percepção e o domínio na prática do desenho. Durante o semestre, foram aplicadas as atividades principais que foram definidas segundo as capacidades de percepção apresentadas por Edwards (2002):

I. Percepção das bordas (contornos);

II. Percepção dos espaços (áreas e superfície);

III. Percepção das relações entre partes (componentes e proporções);

IV. Percepção das luzes e da sombra (volume ou tridimensionalidade);

V. Percepção do todo (Gestalt).

Segundo a autora, tais capacidades de percepção são as habilidades básicas para representação do real através do desenho, formando uma habilidade global (Edwards, 2002. p. 18). Desse modo, as cinco atividades principais avaliativas aplicadas aos alunos de design, distribuídas pelo semestre letivo, foram:

I. Entendimento das formas geométricas; II. Ampliação e redução; 
Procesos Urbanos N 4 Enero - Diciembre; 2017
III. Perspectiva;
IV. Luz e sombra;
V. Pontilhismo (granido).

Para a atividade I, os alunos relembraram os conceitos de formas geométricas e foram introduzidos nas instruções sobre desconstrução na observação de um objeto. Foram apresentados diversos objetos para serem desconstruídos graficamente em quadrados, círculos, triângulos, cubos, cilindros, cones, pirâmides e outras formas geométricas. Assim, conseguiam compreender que a forma do objeto poderia ser retratada graficamente pela construção por etapas, observando seus contornos, suas áreas e superfícies. A atividade prática avaliativa consistiu em desenhar linearmente um conjunto de objetos cúbicos e cilíndricos com lápis 2B, como caixas e garrafas.

Para o desenvolvimento da atividade II, foi apresentado o conceito de malha (ou grade) como recurso para ampliação e redução na execução de desenhos. Esse artifício permitiu facilitar a observação de pontos de referência na estrutura do objeto a ser desenhando. Os alunos escolheram imagens impressas oriundas de revistas e desenharam com lápis 2B tanto a versão reduzida quanto a versão ampliada da referência.

A atividade III carecia de instruções específicas sobre tipos de perspectiva, linha do horizonte e pontos de fuga. Apesar de estarem cientes dos conceitos básicos também apresentados na disciplina que aborda o desenho técnico, foi importante mostrar o valor da perspectiva no desenho artístico. A partir das orientações, os alunos desenvolveram a atividade em duas etapas utilizando lápis 2B. A primeira solicitou a construção de blocos simplificados em pelo menos três ângulos de perspectiva diferentes, dentro de um mesmo ambiente. $\mathrm{Na}$ segunda etapa (figura 1), os alunos foram para o pátio da instituição observar ambientes e retratar parte deles de acordo com a perspectiva que estavam observando, sem a necessidade de extremo realismo.

Para a atividade IV, os conceitos sobre luz e sombra para adicionar volume aos desenhos foram apresentados, assim como orientações sobre posicionamento de luz e sombra projetada. Os alunos testaram em figuras simples a capacidade de criar diferentes tons com o lápis de desenho 2B, $4 B$ e $6 B$. Depois desenvolveram a atividade ao desenhar uma composição de objetos sob a luz de uma luminária, buscando um resultado mais realista.

Finalmente, na atividade $V$, os alunos foram orientados a utilizar seus conhecimentos em luz e sombra, dessa vez para trabalhar com a técnica de granido (ou stippling), um tipo de pontilhismo que utiliza apenas pequenos pontos de uma única cor (Deussen; Isenberg. 2013). As instruções foram transmitidas para a turma através de exemplos e comparações entre referências e seus desenhos feitos com pontos. Essa atividade foi desenvolvida com lápis $2 \mathrm{~B}$ para esboço e caneta nanquim descartável $0.2 \mathrm{~mm}$ para finalização. A referência foi um balão de festa inflado da cor preta que foi cedido a cada aluno e fixado nas mesas com fita adesiva.

Após todas as atividades, no final do semestre letivo, foi solicitado aos alunos que trouxessem o mesmo objeto desenhando no início da disciplina para fazer uma nova representação. Como citado, o intuito foi verificar as capacidades adquiridas com as atividades que trabalharam alguns conceitos importantes para o desenho de observação.

\section{Resultados}

A cada atividade desenvolvida pelos alunos, foi percebida a melhoria nas técnicas de representação e no resultado final dos desenhos, mesmo nas primeiras atividades. Com a aplicação das instruções teóricas, os alunos puderam entender os procedimentos necessários, algumas vezes desconhecidos por eles, para a realização adequada de um desenho de observação. Também foi perceptível a satisfação de cada aluno com seus resultados, mesmo havendo diferentes curvas de aprendizado. A comparação entre o primeiro e o último desenho realizado na disciplina evidencia a evolução dos alunos, mostrando melhor enquadramento e uso das técnicas aprendidas (figuras 2 - 3). As orientações sobre formas geométricas, perspectiva, luz e sombra assim como os 
treinos práticos com os exercícios propostos, aparentaram ser bastante relevantes na melhoria da capacidade representativa.

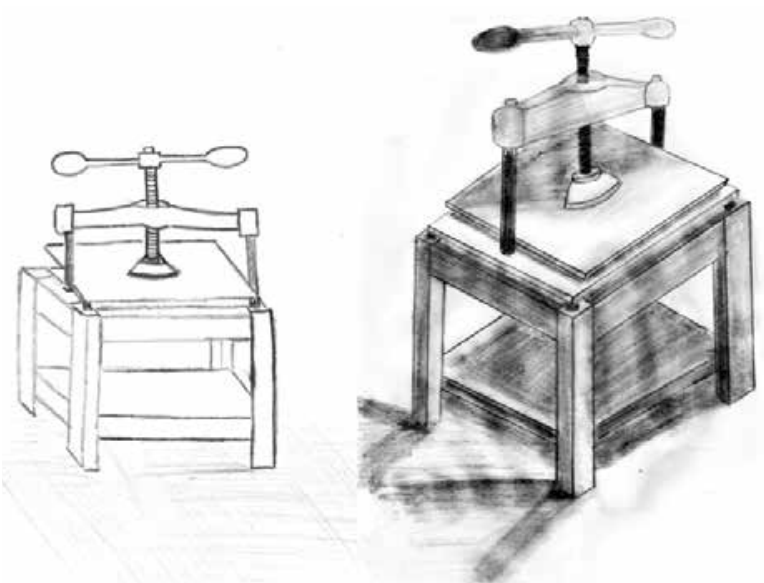

Figura 1. Prensa manual, desenhada pelo aluno $\mathrm{A}$. Percebe-se o melhor uso da perspectiva e do volume. Fonte: o autor:

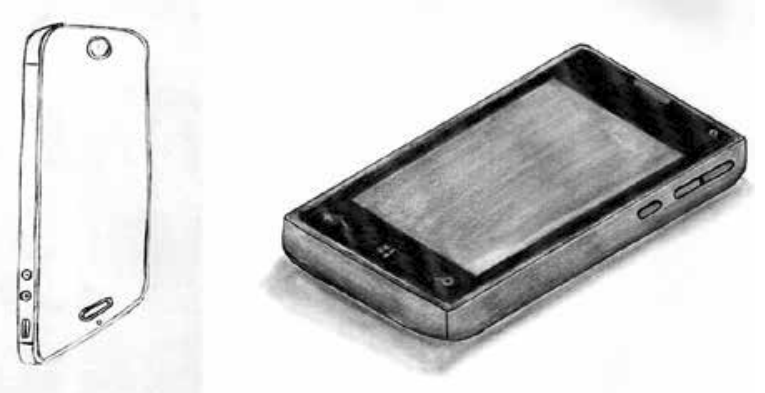

Figura 2. O mesmo aparelho desenhado novamente pelo aluno $B$, com um novo ângulo evidencia detalhes e diferencia melhor os elementos do objeto. Fonte: do autor.
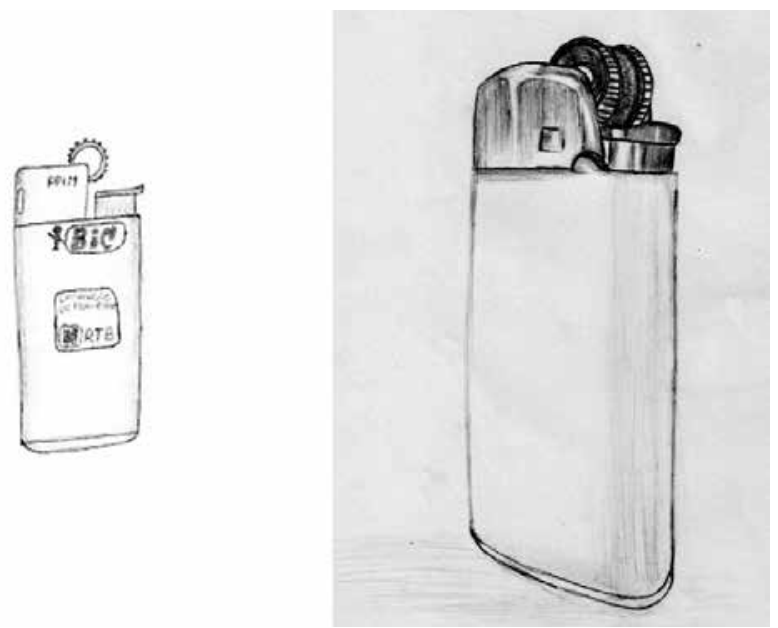

Figura 3. As representações do objeto feitas pelo aluno $C$ indicam a melhoria em sua capacidade de desenho. Fonte: do autor.

\section{Uma abordagem avaliativa de métodos e técnicas}

Em idade adulta, uma pessoa normalmente já possui alguma habilidade de desenho, pois já passou por etapas específicas durante sua infância e adolescência, que ensinaram a prática de algum modo. Independente do nível de habilidade para desenhar, acredita-se que há métodos que melhoram o desempenho de quem desenha. Dentro do espectro profissional, a importância está em formar indivíduos com controle sobre as suas criações, expressando visualmente suas ideias, com as devidas formas e proporções. O desenho e seu domínio se torna um modo de se instrumentalizar (Nakata, 2010. p. $55)$. Nesse capítulo o autor relata parte de sua pesquisa de mestrado que pretende avaliar métodos e técnicas para a melhoria do ensino do desenho de observação na graduação em Design.

Acredita-se que algumas metodologias no ensino do desenho, assim como algumas técnicas, permitem que os alunos tenham melhor capacidade produtiva, beneficiando-os mesmo durante a graduação. Após a conclusão do curso, eles seriam mais aptos e seguros no desenvolvimento e apresentação de projetos profissionais. A pesquisa experimental, em andamento, propõe contribuir com o ensino e a prática do desenho, assim como no entendimento das dificuldades de representação através de desenhos feitos por alunos de curso superior. De modo dedutivo, pretende-se sugerir os dados levantados como parâmetros para atividades em disciplinas de desenho de observação.

Perceber que um objeto, com sua forma peculiar, pode ser entendido melhor como uma junção de algumas formas geométricas, pode proporcionar uma visão diferenciada e um desenho com proporções mais reais. Além disso, pontos de referências visuais também podem auxiliar na construção gráfica de um desenho. O dispositivo de Albrecht Dürer, que auxiliava o artista na reprodução de um modelo por uma grade de marcações lineares horizontais e verticais, ilustra bem esse tipo de recurso (Edwards, 2002. p. 163). Perceber o contorno do que é observado e destacar os espaços 
negativos e positivos também é um método para compreender visualmente o que se vê para que possa ser desenhado com mais precisão. Desse modo, a pesquisa utilizou essas técnicas e recursos auxiliares para definir um método auxiliar denominado aqui como Geometria, Grade e Desconstrução.

Por sua vez, o desenho de observação utilizando-se da técnica de granido para aplicação de pequenos pontos que simulam volume, diferentes tons e textura, permite - estímulo da observação e retratação de detalhes, aprimorando o senso de percepção (Nakata, 2011. p. 120). Acredita-se que essa técnica tem potencial para extrair um melhor resultado no desenho, principalmente na definição de volume. Nesse trabalho, classificamos essa metodologia como Granido.

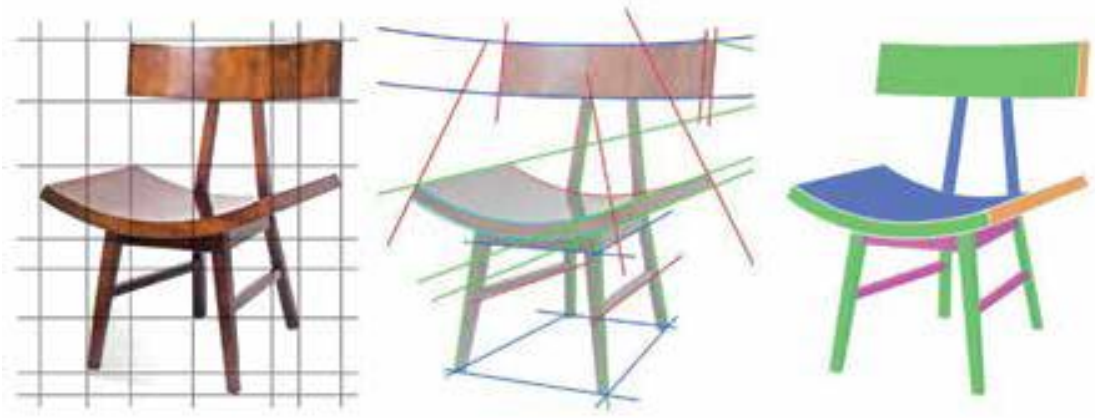

Figura 4. Exemplo de metodologia aplicada sobre imagem de referência para evidenciar formas e proporções. Fonte: 0 autor.

Com base nos recursos auxiliares citados, a pesquisa definiu esses dois métodos para o desenho de observação a serem verificados quanto a sua influência no aprendizado e no resultado da atividade (figura 5). A hipótese é que esses métodos podem contribuir fortemente para o entendimento e melhoria da prática do desenho. Foi definida então uma amostra que contém alunos matriculados em um curso de graduação de Bacharel em Design e ex-alunos recém-formados do mesmo curso. Parte dessa amostra são os mesmos indivíduos que o capítulo anterior apresentou.

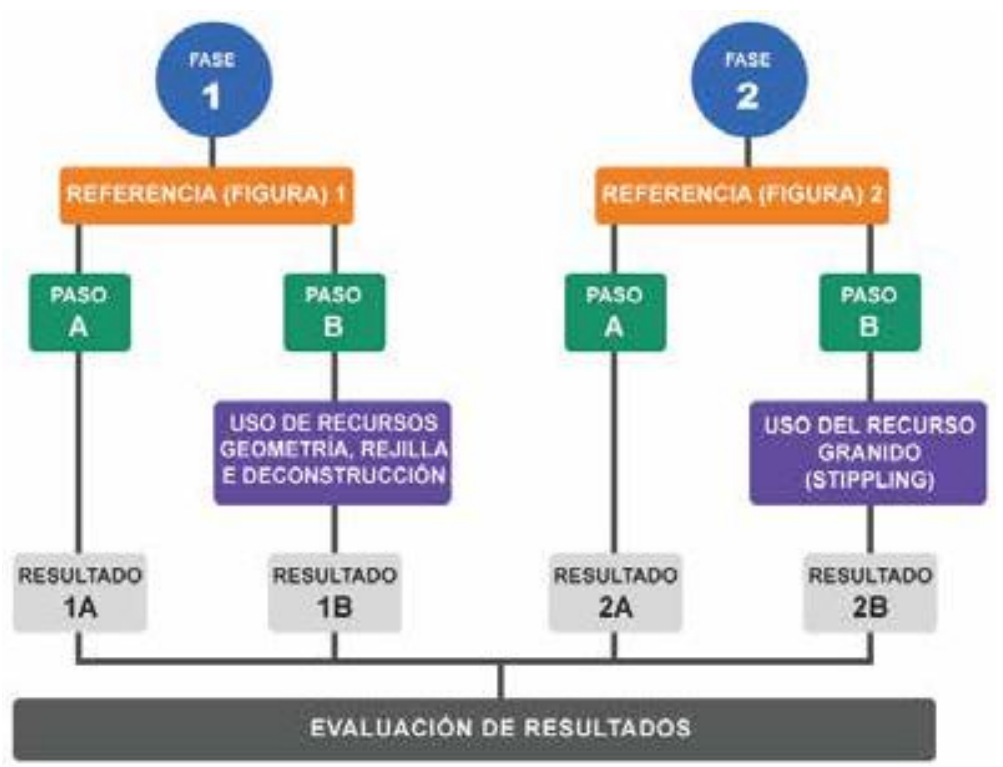

Figura 5. Método e técnicas aplicadas na pesquisa. Fonte: do autor 
Inicialmente, foram coletados dados da amostra que traçam seu perfil quanto ao domínio do desenho e experiência com a prática. Depois, a avaliação das técnicas e a coleta de dados consistem em quatro etapas. Nas etapas $1 \mathrm{~A}$ e $1 \mathrm{~B}$ os indivíduos deveriam representar linearmente, em folhas de sulfite A4 com lápis de desenho 2B sem o uso de ferramentas de precisão como régua e compasso, um objeto utilizando uma referência impressa. $\mathrm{Na}$ etapa $1 \mathrm{~A}$ deveriam desenhar usando seus conhecimentos e técnicas, sem instruções adicionais do pesquisador. $\mathrm{Na}$ etapa $1 \mathrm{~B}$, 0 mesmo objeto deveria ser representado, mas dessa vez seguindo instruções que o pesquisador cedia (figura 4). Nessa primeira fase, o objetivo é verificar a representação correta de proporções e forma com o auxílio de linhas-guias e entendimento das partes de cada objeto.

Nas etapas $2 \mathrm{~A}$ e $2 \mathrm{~B}$, o mesmo procedimento está sendo seguido com a representação de um novo objeto. No entanto, o objetivo é avaliar a representação de detalhes e de volume aplicado no desenho do objeto. $\mathrm{Na}$ etapa $2 \mathrm{~A}$, os indivíduos precisam desenhar retratando o volume e textura que conseguem observar na figura de referência. Na etapa 2B, a mesma referência será usada para a reprodução do objeto com o uso do desenho pontilhado.

No final das etapas $1 \mathrm{~B}$ e $2 \mathrm{~B}$, um formulário é cedido aos indivíduos para que eles descrevam sua experiência e satisfação com as técnicas cedidas pelo pesquisador e com os resultados obtidos. Portanto, todos os desenhos também passam por avaliação do pesquisador e dos indivíduos da amostra. Um grupo de outros avaliadores também classificará os desenhos, sob uma ótica de qualidade técnica, comparados à sua referência. Porém, estes avaliadores não saberão da ordem em que foram feitos. A ordenação de todos os dados coletados pretende classificar o grau de influência das instruções e das técnicas aplicadas. Com isso, objetiva-se contribuir com 0 ensino do desenho, em especial em cursos de graduação de design.

\section{Considerações finais}

Esse trabalho objetivou apresentar informações sobre o ensino do desenho por meio de atividades realizadas na disciplina de desenho de observação em um curso superior de design. Inclui ainda os dados parciais de uma pesquisa que também aborda as atividades dessa disciplina. 0 texto procurou apontar as deficiências no ato desenhar e ações que buscaram auxiliar a retomada da atividade.

Considera-se importante a condução e mediação pelo professor no modo como as instruções são direcionadas aos alunos. Cabe entender o perfil desse grupo e suas necessidades dentro do âmbito universitário e, posteriormente, profissional. A experiência com alunos de design se mostrou satisfatória com resultados que demonstraram a evolução no ato de observar e retratar algo utilizando o desenho.

Os resultados parciais da pesquisa que está avaliando métodos e técnicas auxiliares para o desenho de observação se mostram promissores, mesmo com indivíduos que declaram ter maior aptidão com o desenho manual. Procurou-se listar e condensar recursos de fácil entendimento e que ajudam na percepção e na execução do desenho. Em trabalhos futuros, pretendese continuar levantando mais informações sobre métodos utilizados no ensino do desenho de observação para contribuir na prática docência e na capacidade dos futuros profissionais de design, arquitetura e moda. 


\section{Referências}

Deussen, O.; Isenberg, T. (2013) Halftoning and Stippling. In Paul Rosin and John Collomosse, Image and Video based Artistic Stylisation, volume 42 of Computational Imaging and Vision, chapter 3, p. 45-61. Springer, London, Heidelberg. Disponível em <https://tobias.isenberg. cc/personal/papers/Deussen_2013_HS.pdf>. Acesso em 17 de julho de 2017.

Edwards, B. (2002) Desenhando com o lado direito do cérebro. Rio de Janeiro: Editora Ediouro. 299 p.

Jenny, P. (2014) Técnicas de desenho. São Paulo: Gustavo Gili. 167 p.

Massironi, M. (2015) Ver pelo desenho: aspectos técnicos, cognitivos, comunicativos. Lisboa: Edições 70. 201 p.

Nakata, M. K; Silva, J. C P. (2011) Desenho para design. 1.ed. Bauru: Canal 6 Editora. 160 p.

Nakata, M. K. et al. (2010) Ensaios em design: arte, ciência e tecnologia. 1.ed. Bauru: Canal 6 Editora. 208 p.

Nakata, M. K. et al. (2011) Ensaios em design: ensino e produção de conhecimento. 1. ed. Bauru: Canal 6 Editora. 192 p.

Agradecimentos

Os autores agradecem a CAPES pelo apoio financeiro destinado à pesquisa experimental em andamento, descrita no capítulo 4. 